\title{
Dynamic Characteristics of a Cantilever Beam with Transverse Cracks
}

\author{
R. K. Behera and D. R. K. Parhi \\ Department of Mechanical Engineering, National Institute of Technology, Rourkela, 769008, Orissa, India
}

\section{S. K. Sahu}

Department of Civil Engineering, National Institute of Technology, Rourkela, 769008, Orissa, India

(Received 20 April 2004; accepted 28 June 2005)

\begin{abstract}
It has long been observed that the dynamic response of a structure changes due to the presence of a crack. Scientific analysis of such phenomena can be utilised for fault diagnosis and the detection of cracks in structures. The present investigation is an attempt in that direction. Theoretical expressions have been developed in order to determine the natural frequencies and mode shapes for an elastic cantilever beam with two cracks using flexibility influence coefficients and a local stiffness matrix. The numerical results for the beams without cracks, with one crack, and with two cracks are compared. It has been observed from the numerical results that there are appreciable changes in the vibration characteristics of the cantilever beam with and without cracks. This method can be utilised for multi crack identification of structures.
\end{abstract}

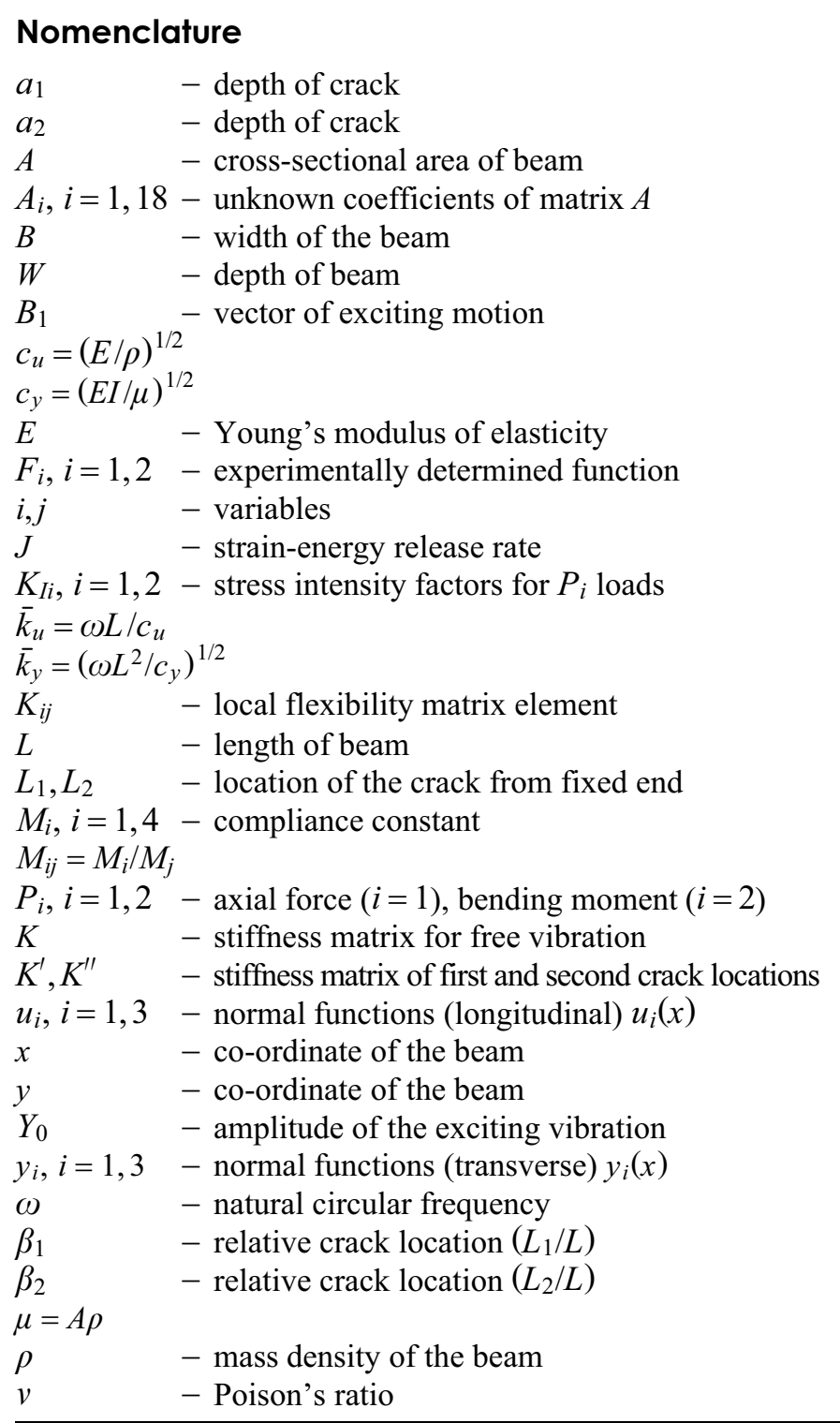

